\title{
Crenças em saúde para o controle da hipertensão arterial
}

\author{
H ealth beliefs for the control of arterial hypertension
}

Cláudia Geovana da Silva Pires ${ }^{1}$

Fernanda Carneiro M ussi ${ }^{1}$

${ }^{1}$ Escola de Enfermagem, UFBA, H ospital São Rafael. Rua Renato M endonça, Ed. Camélia, apto. 401 Brotas. $40285-440$ Salvador BA. cgspires@uol.com.br
Abstract $\mathrm{H}$ ealth beliefs can interfere with the adherence to arterial hypertension therapy. The aim of this descriptive exploratory study that adopted the $\mathrm{M}$ odel of $\mathrm{H}$ ealth Beliefs as a theoretical referencewas to estimate percentages of health beliefs about the benefits of prevention and control measures of arterial hypertension and to identify thesocial-demographic factors associated with these beliefs. Thestudy was conducted in a $\mathrm{H}$ ealth Center in the city of Salvador, with 106 adults self- declared as black, and with a medical diagnosis of arterial hypertension. For the interviews we used a "Scale of $\mathrm{H}$ ealth Beliefs" about 13 behaviors related to disease prevention and control measures. The data analysis was based on percentage rates, frequency of cases and scores and the social-demographic factors associated to these beliefs wereanalyzed based on the prevalencerate. The global analysis showed predominance in the category "beliefs about benefits" for 12 behaviors. $M$ en and women realized different benefits from these behaviors. The socio-economically less favored strata, young adults and individuals living without a partner tended to perceive less benefits from the prevention and control measures of arterial hypertension.

Key words Hypertension, Risk factors, Adherence, Education in health, Nursing
Resumo As crenças em saúde podem influenciar na adesão ao tratamento da hipertensão arterial. Estimar o percentual de crenças em saúde sobre barreiras e benefícios quanto às medidas de prevenção e controle da hipertensão arterial e conhecer os fatores sociodemográficos associados a essas crenças foi 0 objetivo deste estudo descritivo-exploratório, que adotou como referencial teórico 0 M odelo de Crenças em Saúde. Foi realizado em um centro de saúdeem Salvador, com 106 adultos, autodeclarados negros e com diagnóstico médico de hipertensão arterial. Para a entrevista, utilizou-se uma Escala de Crenças em Saúde sobretre zecomportamentos relacionadosàs medidas depre venção e controle da doença. Para análise dos dados, utilizaram-se índices percentuais, freqüência de casos, escores e a razão de prevalência. O stestes estatísticos foram verificados no nível de $5 \%$ de significância. A análise global mostrou predomínio da categoria "crenças sobre benefícios" para doze com portamentos de saúde. Homens emulheres perceberam diferentemente benefícios para esses comportamentos e constatou-se uma tendência à percepção de menos benefícios quanto às me didas de prevenção e controle da hi pertensão arterial em estratos socioeconômicos menos favorecidos, adultos joven se pessoas sem companhei ro.

Palavras-chave Hipertensão, Fatores de risco, Adesão, Educação em saúde, Enfermagem 
Introdução

A hipertensão arterial constitui-se em relevante problema de saúde pública, ressaltando-se que apresenta el evado índice de prevalência emortalidade'; 'é uma doença multifatorial, desencadeada deforma isolada ou está associada ao agravamento de diversas doenças'; ; em virtude do seu caráter crônico e incapacitante, gera aposentadorias precoces, longo período de internação, alto custo para o tratamento, alterações na auto-estima e autoconceito do indivíduo ${ }^{2}$; aliado ao discurso disciplinador, a pessoa hipertensa apresenta uma condição de vida cerceada, necessitando de monitoramentos constantes, mudan ça de hábitos devida, incluindo a medicalização da alimentação, da atividade física'; o patamar de adesão é insatisfatório, contribuindo para a morbimortalidade da doença ${ }^{2,3}$. Esse panorama revela que a prevenção e o controle da doença são imprescindíveis e um desafio para profissionais de saúde, autoridades públicas e indivíduos hipertensos.

A adesão ao tratamento da hipertensão arterial vem sendo considerada um processo comportamental complexo, fortemente influenciado pelo meio ambiente, pelo indivíduo, pelos profissionais de saúde e pel os cuidados de assistência médica ${ }^{3}$ e perpassado por dimensões biológicas, socioeconômicas, psicológicas e culturais.

A análise da literatura mostra que os fatores que interferem na adesão estão associados à terapêutica, ao sistema de saúde e ao indivíduo ${ }^{46}$. Apesar da importância de se estudar essa gama defatores, pouco tem sido explorado sobre aqueles associados às pessoas hipertensas, mais especificamente da raça/cor negra, no quese refere às suas crenças em saúde sobre as medidas de prevenção e controle da doença, a saber: dieta hipossódica e com baixo teor de gordura saturada e colesterol, abandono do tabagismo, redução do consumo deálcool, prática deatividadefísica, lazer, relaxamento, manutenção do peso corporal ideal, controle da pressão arterial e comparecimento periódico às consultas médicas.

Entende-se que a mudança no estilo de vida relaciona-se às crenças e aos comportamentos apreendidos e incorporados pelas pessoas na convivência social ${ }^{7}$. Portanto, é inegável que 0 controle da pressão arterial não se limita apenas à abordagem do corpo doente, sendo necessário considerar a experiência de vida ea subjetividade como aspectos imprescindíveis no processo de adoecer e cuidar de si. Conhecer as crenças em saúde sobre as medidas de controle da hipertensão arterial possibilita mostrar indicadores de adesão ao tratamento, identificar grupos de risco e repensar as formas de cuidado à saúde.

\section{Objetivos}

Estimar o percentual de crenças em saúde sobre barreiras e benefícios quanto às medidas de prevenção e controle da hipertensão arterial para pessoas negras e conhecer os fatores sociodemográficos associados a essas crenças.

\section{Basesteórico-metodológicas}

Tipo de estudo

Esta é uma pesquisa descritiva, exploratória, de natureza quantitativa.

Modelo de Crenças em Saúde

No início dos anos 1950, um grupo de psicólogos do Serviço de Saúde Pública dos Estados Unidos desenvolveu este modelo, na tentativa de explicar o motivo pelo qual as pessoas não se preveniam adequadamente contra certas doenças. Para explicar a ação preventiva, o modelo foi publicado por Rosenstock, em 1966, e aplicado a comportamentos relacionados à manuten ção da saúdeem geral ${ }^{8}$. Tem como premissa que o mundo perceptual da pessoa determina o seu comportamento, não o ambiente físico ${ }^{9}$.

O M odelo de Crenças em Saúde, em relação ao comportamento preventivo, é demonstrado por quatro dimensões ou variáveis, assim colocadas:

a) susceptibilidade percebida - refere-se à percepção subjetiva do risco de a pessoa contrair determinada condição ou doença;

b) severidade percebida - o grau de severidade da doença é avaliado tanto pelo grau de estimulação emocional criado em torno dela como pelas conseqüências biológicas, sociais, emocionais e financeiras que poderá acarretar;

c) benefícios percebidos - referem-se à crença na efetividade da ação ena sua percepção, com resultados positivos;

d) barreiras percebidas - os aspectos negativos da ação são avaliados em uma análise do tipo custo-benefício, considerando-se os esforços empreendidos, a caracterização invasiva dos procedimentos diagnósticos e terapêuticos, as vivências, os aborrecimentos e a dor, que servem como barreiras para a ação e estimulam moti- 
vos conflitantes de enfrentamento. 0 potencial para ação resulta da combinação das variáveis susceptibilidade e severidade percebidas na doença, en quanto a modalidade de ação éescolhida em função da percepção dos benefícios, excluindo-se as barreiras percebi das nos comportamentos em saúde?

\section{Lócus do estudo}

A pesquisa foi realizada num centro de saúde do município de Salvador, situado no bairro da Liberdade, que abriga no seu contingente populacional $82,8 \%$ de pessoas da cor preta e parda ${ }^{10}$ e serve de referência para 0 atendimento de pessoas com hipertensão arterial.

\section{Amostra}

A amostra constituiu-se de 106 adultos, de ambos os sexos, com diagnóstico médico de hipertensão arterial há no mínimo seis meses, que se autodeclararam da cor preta e/ ou parda, compareciam ao local de estudo para consulta e/ou verificação da pressão arterial e estavam cadastradas no programa de hipertensão. Para o cálculo do tamanho da amostra, embasado em Rosner ${ }^{11}$, assumiu-se a freqüência esperada de benefícios de $50 \%$, com um erro aceitável de $40 \%$, nível de significância de $5 \%$ e poder da amostra em torno de $60 \%$. 0 recorte racial justifica-se pela possibilidade de haver al guma especificidade das crenças relacionadas às pessoas de cor/ raça negra, considerando as suas origens, os processos de escravidão e exclusão social, as condições socioculturais e financeiras e outras formas de vivência excludente, fatores que certamente exercem significativa influência nos comportamentos de saúde. A prevalência e determinantes da hipertensão arterial nas pessoas negras e 0 fato de a cidade de Salvador possuir o maior número de afrodescendentes fora da África justificam também privilegiar as crenças junto a esse grupo racial.

\section{Coleta de dados}

A Parte I do formulário foi constituída por questões fechadas sobre dados de caracterização dos participantes ea Partell, pela“"Escala deCrenças" extraída da tese de doutorado de Dela Coleta ${ }^{8}$, após obtenção da sua autorização para uso nesta pesquisa. A escala ${ }^{8}$ apresenta sentenças sobre crenças de barreiras e benefícios relacionados a cada um dos treze comportamentos de saúde referentes às medidas de prevenção e controle da hipertensão arterial, as quais constam em anexo.

$\mathrm{Na}$ apresentação da escala, para cada comportamento de saúde, aos itens (sentenças) sobre "benefícios percebidos" foram intercalados os itens sobre "barreiras percebidas" 8 . 0 levantamento das crenças sobre cada comportamento de saúde era precedido da pergunta: Qual a sua opinião sobre...? O Quadro 1 éilustrativo.

A escala éapresentada no formato Likert, com cinco níveis de resposta, variando de DT (discordo totalmente=1), D (discordo=2), I (indeciso=3), $C($ concordo $=4)$ até $C T$ ( concordo totalmente $=5)$. Com base no M odelo de Crenças em Saúde, para cada sentença, níveis de resposta que evidenciam resultados positivos para o comportamento em saúde são considerados benefícios eníveis de resposta que evidenciam aspectos negativos para 0 comportamento em saúde, podendo ser impeditivos para ação, são considerados barreiras.

Cada participante foi orientado sobre os cinco níveis de resposta. Para facilitar a resposta, foi criada uma figura, para que pontuasse a sua resposta à medida que as frases fossem lidas, na qual as cores verde, azul, rosa, amarela e vermeIha correspondiam a cada nível de resposta.

Quadro 1. Crenças em saúde sobre o uso de pouco sal na comida.

\begin{tabular}{|l|l|l|l|l|l|}
\hline 1. Qual a sua opinião sobre usar pouco sal na comida? & DT =1 & D =2 & I=3 & C =4 & CT=5 \\
\hline $\begin{array}{l}\text { BEN SAL -Deve-se usar pouco sal na comida porque ele aumenta } \\
\text { a pressão arterial }\end{array}$ & & & & & \\
\hline BARSAL -A comida com pouco sal não tem gosto & & & & & \\
\hline
\end{tabular}


A coleta de dados foi realizada de julho a se tembro de 2006. Os participantes foram escolhidos de forma al eatória, enquanto aguardavam a verificação da pressão arterial ou a consulta pela equipe multidisciplinar. As pessoas que chegavam primeiro eram abordadas e caso se identificassem como da cor preta e/ou parda e tivessem diagnóstico médico dehipertensão arterial há pelo menos seis meses, confirmado pelo cartão de registro no Centro deSaúde, fazia-seo convitepara participar da pesquisa; a seguir, recebiam explicações sobre seus objetivos. Em caso de aquiescência, a pessoa era encaminhada para uma sala privativa e, após leitura, esclarecimento eassinatura do termo de consentimento livre e esclarecido, a entrevista era iniciada e gravada. Ao término, agradecia-se ao entrevistado pela contribuição à pesquisa. Aos participantes foram assegurados o sigilo da identidade pessoal, o direito de abandonar o estudo a qualquer momento e a privacidade $^{12}$. 0 estudo obteve aprovação do Comitêde Ética e Pesquisa da Secretaria Estadual de Saúde da Bahia.

Tratamento e análise dos dados

As entrevistas gravadas foram digitadas nos formulários, em outro momento. Os dados foram codificados, digitados e processados no SPSS (Statistical Package of Social Science) for Windows.

Os dados de caracterização da amostra foram analisados com base em números absolutos e percentuais.

Para análise do percentual de crenças sobre barreiras e benefícios relacionados às treze medidas de prevenção e controle da hipertensão arterial, procedeu-se a construção do indicador sobre crenças, da seguinte maneira: a) para cada participante do estudo e para cada uma das treze medidas de preven ção e controle que constituem a escala de crenças, criou-se uma média aritmética para barreiras, utilizando-se os itens (sentenças) correspondentes às barreiras, e uma média aritmética para benefícios, utilizando-se os itens (sentenças) correspondentesa benefícios; $b$ ) estabeleceu-sea diferença entrea média aritmética da barreira e a média aritmética de benefício, construídas como descrito em "a", para cada uma das treze medidas de prevenção e controle da escala de crenças e para cada participante da pesquisa; c) os resultados da diferença entre as médias aritméticas de cada participante construídas como descrito em " $b$ " foram classificados em três categorias: 1 ) crenças sobre benefícios = valores da dife- rença das médias aritméticas abaixo de zero; 2) crenças sobre barreiras $=$ valores da diferença das médias aritméticas acima de zero; 3 ) indecisos na percepção de crenças sobre barreiras e ben efícios = val ores da diferença das médias aritméticas igual a zero, ou seja, não predominaram crenças sobre barreiras ou benefícios. Posteriormente, as treze medidas de prevenção e controle foram associadas às três categorias do indicador crenças, para verificação da distribuição percentual.

Tomando-se como exemplo uma classificação hipotética das respostas de um participante para as sentenças relativas às crenças sobre "manter o peso ideal para idade ealtura", ilustra-se, no Quadro 2, como foi estimado o percentual de crenças sobre benefícios e barreiras para esse comportamento em saúde.

0 trabalho com os dados obedeceu às seguintes etapas:

a) somando-se os valores obtidos para as sentenças benefícios, obteve-se a pontuação 27 ; para as sentenças barreiras, a pontuação 12. Dividindo-se 27 por 7, ou seja, a pontuação total encontrada para benefícios (27) pelo número total de sentenças sobre benefícios (7), chegouseà média aritmética de ben efícios de 3,85. Dividindo-se 12 por 5 , ou seja, a pontuação total encontrada para barreiras (12) pelo número total de sentenças sobre barreiras (5), chegou-se a média aritmética de barreiras de 2,4.

b) na segunda etapa, estabeleceu-se a diferença entre a média aritmética para barreira e a média aritmética para ben efício. Assim, com base no exemplo, a diferença entreas médias foi: 2,4 $3,85=-1,45$.

c) na terceira fase, o resultado da diferença entreas médias aritméticas do participante, construídas como descrito em "b", foi classificado em uma das três categorias descritas anteriormente. Com base no valor obtido de - 1,45, o participante em questão foi classificado na categoria "crenças em saúde sobre benefícios" (valores da diferença das médias aritméticas abaixo dezero). Chegando-se a essa classificação, depreendeu-se que houve predomínio de maior percepção de benefícios para esse participante com relação ao comportamento de saúde analisado.

Para as análises bivariadas, verificou-se a associação dos fatores idade, sexo, renda familiar, escolaridade e estado civil ao indicador crença (categoria crenças sobre benefícios) utilizandose a razão de prevalência (RP) e o intervalo de confiança de $95 \%$. Valores de RP maiores que um e menores que um representaram, respectivamente, maior e menor percepção de benefí- 
Quadro 2. Crenças em saúde sobre manter o peso ideal para idade e altura.

\begin{tabular}{|c|c|c|c|c|c|}
\hline Qual a sua opinião sobre manter o peso ideal para a idade e a altura? & DT $=1$ & $D=2$ & $I=3$ & $C=4$ & $\mathrm{CT}=5$ \\
\hline \multicolumn{6}{|l|}{$\begin{array}{l}\text { BENPESO - Com o peso adequado a pessoa tem mais disposição } \\
\text { e bem-estar }\end{array}$} \\
\hline \multicolumn{6}{|l|}{ BARPESO- Perder peso é um sacrifício } \\
\hline \multicolumn{6}{|l|}{ BEN PESO- 0 excesso de peso faz mal à saúde } \\
\hline \multicolumn{6}{|l|}{ BARPESO- Emagrecer é muito difícil } \\
\hline \multicolumn{6}{|l|}{$\begin{array}{l}\text { BEN PESO- O excesso de peso atrapalha os movimentos e a agilidade da } \\
\text { pessoa }\end{array}$} \\
\hline \multicolumn{6}{|l|}{$\begin{array}{l}\text { BARPESO- É melhor comer à vontade do que ficar se preocupando com } \\
\text { o peso }\end{array}$} \\
\hline \multicolumn{6}{|l|}{ BEN PESO- Quem está acima do peso pode ter problemas de coração } \\
\hline \multicolumn{6}{|l|}{$\begin{array}{l}\text { BARPESO - A pessoa que não faz sua própria comida não pode escolher o } \\
\text { que comer }\end{array}$} \\
\hline \multicolumn{6}{|l|}{ BARPESO- Não acho importante manter o peso } \\
\hline \multicolumn{6}{|l|}{ BEN PESO- É bom controlar o peso para manter a forma, ficar elegante } \\
\hline \multicolumn{6}{|l|}{ BEN PESO- O gordo tem problemas de coluna } \\
\hline BENPESO- A obesidade é uma doença & & & & & \\
\hline
\end{tabular}

cios. Quando o intervalo de confiança inclui a unidade (1), o nível de significância é considerado acima de $5 \%$; quando não inclui, é menor ou igual a 5\% de significância. Todos os testes estatísticos constantes dos resultados foram verificados em nível de $5 \%$ designificância.

\section{Resultados}

D os 106 participantes entrevistados, mais de 2/3 eram mulheres $(73,6 \%)$. A idade mediana foi de 54 anos. Os 50\% dos participantes com idade maisjovem encontravam-senas faixas etárias $<45$ $(24,5 \%)$ e 45 a $54(24,5 \%)$. Ainda, $51 \%$ dos mais velhos, isto é, com idade $\geq 54$ encontravam-sena faixa etária de 54 a 63 anos (26,5\%); de 63 a 69 anos ( $16 \%$ ) e apenas nove pessoas estavam com idade $\geq$ a 70 anos (8,5\%). A presentaram predominantemente baixa escolaridade (até o $1^{\circ}$ grau - 63,2\%) e baixa renda familiar, menor que um salário mínimo (52,8\%). Mais da metade dos participantes exercia atividade profissional, incluindo o trabal ho doméstico (67,0\%) evivia sem companheiro (61,3\%). Predominou a religião católica (69,8\%). A grande maioria recebeu o diagnóstico médico de hipertensão arterial há mais de um ano $(80,2 \%)$ e estava em tratamento medicamentoso e/ou não medicamentoso (87,7\%).
A análise global sobre as crenças em saúde quanto às medidas de prevenção e controle da hipertensão arterial mostrou predomínio da categoria "crenças em saúde sobre benefícios" para dozemedidas de prevenção e controle da doença, excetuando-se para o comportamento evitar preocupação. Ainda é relevante considerar que quase $1 / 3$ da amostra percebeu barreiras para usar menos sal.

As Tabelas de 1 a 5 mostram a análise dos fatores sociodemográficos associados às percepções de crenças em saúde sobre benefícios, segundo as doze medidas de controle da hipertensão arterial. N ota-sequehomens emulheres perceberam de modo diferente maior ou menor benefício quanto aos dozecomportamentos de saúde, mas essa diferença não foi estatisticamente significante. Observou-se que as pessoas com menos de 45 anos perceberam mais benefícios quando comparadas àquelas na faixa etária e" 54 anos apenas para fazer relaxamento, ter horas de diversão e lazer e praticar exercícios físicos. Pessoas com até 010 grau, em relação àquelas com 3o grau, perceberam menos benefícios em nove comportamentos de saúde, exceto para manter peso ideal e evitar o consumo de álcool, havendo diferença estatisticamente significante parafazer relaxamento econsultar o médico uma vez por ano. 
Tabela 1. Fatores sociodemográficos associados à categoria crenças sobre benefícios em relação à dieta. Salvador, Bahia, 2006. $(n=106)$

\begin{tabular}{|c|c|c|c|c|c|c|c|c|c|c|c|}
\hline \multirow[b]{3}{*}{ Variáveis } & \multicolumn{11}{|c|}{ Dieta } \\
\hline & \multicolumn{4}{|c|}{ Sal } & \multicolumn{4}{|c|}{ Gordura } & \multicolumn{3}{|r|}{ Doce } \\
\hline & $\mathrm{N}$ & $\%$ & $\mathrm{RP}$ & IC95\% & $\mathrm{N}$ & $\%$ & $\mathrm{RP}$ & IC95\% & $\mathrm{N}$ & $\%$ & IC95\% \\
\hline \multicolumn{12}{|l|}{ Gênero } \\
\hline Homens & 28 & 67,9 & 0,97 & $(0,72,1,30)$ & 27 & 77,8 & 0,93 & $(0,75,1,17)$ & 28 & 89,3 & $0,95(0,83,1,10)$ \\
\hline Mulheres & 77 & 70,1 & 1 & & 78 & 83,3 & 1 & & 77 & 93,5 & 1 \\
\hline \multicolumn{12}{|l|}{ Idade(anos) } \\
\hline$<45$ & 25 & 56 & 0,76 & $(0,52,1,10)$ & 25 & 80 & 0,97 & $(0,78,1,21)$ & 26 & 92,3 & $1 \quad(0,88,1,13)$ \\
\hline $45-54$ & 26 & 73,1 & 1,07 & $(0,81,1,41)$ & 26 & 88,5 & 1,11 & $(0,93,1,33)$ & 26 & 96,2 & $1,05(0,95,1,17)$ \\
\hline$>=54$ & 54 & 74,1 & 1 & & 54 & 79,6 & 1 & & 53 & 90,6 & 1 \\
\hline \multicolumn{12}{|l|}{ Estado civil } \\
\hline Sem companheiro & 64 & 68,8 & 0,97 & $(0,75,1,25)$ & 64 & 84,4 & 1,09 & $(0,9,1,31)$ & 65 & 90,8 & $0,95(0,86,1,06)$ \\
\hline Com companheiro & 41 & 70,7 & & & 41 & 78 & & & 40 & 95 & \\
\hline \multicolumn{12}{|l|}{ Escolaridade } \\
\hline A té $1^{\circ}$ grau & 67 & 68,7 & 0,97 & $(0,74,1,25)$ & 67 & 79,1 & 0,91 & $(0,76,1,08)$ & 66 & 92,4 & $1 \quad(0,89,1,12)$ \\
\hline $2^{\circ} \mathrm{grau}$ & 31 & 74,2 & 1,1 & $(0,85,1,42)$ & 31 & 87,1 & 1,09 & $(0,91,1,3)$ & 32 & 93,8 & $1,02(0,91,1,14)$ \\
\hline 3o grau & 7 & 57,1 & & & 7 & 85,7 & & & 7 & 85,7 & \\
\hline \multicolumn{12}{|l|}{ Renda } \\
\hline$<1 \mathrm{s.m}$ & 56 & 66,1 & 0,9 & $(0,7,1,16)$ & 55 & 74,5 & 0,83 & $(0,69,0,99)$ & 55 & 92,7 & $1 \quad(0,90,1,12)$ \\
\hline 1 a 3 s.m & 33 & 75,8 & 1,14 & $(0,88,1,46)$ & 33 & 93,9 & 1,23 & $(1,05,1,44)$ & 33 & 90,9 & $0,98(0,86,1,11)$ \\
\hline$>3 \mathrm{s.m}$ & 16 & 68,8 & & & 17 & 82,4 & & & 17 & 94,1 & \\
\hline
\end{tabular}

RP (Razão de prevalência); IC95\% (Intervalo de confiança) * $p<0,05$

Tabela 2. Fatores sociodemográficos associados à categoria crenças sobre benefícios em relação ao controle médico. Salvador, Bahia, 2006. $(n=106)$

\begin{tabular}{|c|c|c|c|c|c|c|c|c|}
\hline \multirow[b]{3}{*}{ Variáveis } & \multicolumn{8}{|c|}{ Controle médico } \\
\hline & \multicolumn{4}{|c|}{ Consulta médica uma vez/ano } & \multicolumn{4}{|c|}{ Uso do remédio receitado pelo médico } \\
\hline & $\mathrm{N}$ & $\%$ & $\mathrm{RP}$ & IC95\% & $\mathrm{N}$ & $\%$ & $\mathrm{RP}$ & IC95\% \\
\hline \multicolumn{9}{|l|}{ Gênero } \\
\hline Homens & 28 & 96,4 & 1,02 & $(0,93,1,11)$ & 28 & 82,1 & 0,95 & $(0,78,1,16)$ \\
\hline Mulheres & 78 & 94,9 & 1 & & 72 & 86,1 & & \\
\hline \multicolumn{9}{|l|}{ Idade(anos) } \\
\hline$<45$ & 26 & 92,3 & 0,96 & $(0,85,1,08)$ & 25 & 80 & 0,92 & $(0,74,1,14)$ \\
\hline $45-54$ & 26 & 96,2 & 1,01 & $(0,92,1,11)$ & 23 & 91,3 & 1,1 & $(0,93,1,30)$ \\
\hline$>=54$ & 54 & 96,3 & & & 52 & 84,6 & & \\
\hline \multicolumn{9}{|l|}{ Estado civil } \\
\hline Sem companheiro & 65 & 92,3 & 0,92 & $(0,86,0,99)$ & 59 & 83,1 & 0,95 & $(0,80,1,11)$ \\
\hline Com companheiro & 41 & 100 & & & 41 & 87,8 & & \\
\hline \multicolumn{9}{|l|}{ Escolaridade } \\
\hline Até 1o grau & 67 & 92,5 & 0,92 & $(0,86,0,99)$ & 65 & 83,1 & 0,94 & $(0,80,1,10)$ \\
\hline $2^{\circ}$ grau & 32 & 100 & 1,07 & $(1,00,1,14)$ & 29 & 86,2 & 1,02 & $(0,85,1,22)$ \\
\hline 3o grau & 7 & 100 & & & 6 & 100 & & \\
\hline \multicolumn{9}{|l|}{ Renda } \\
\hline$<1$ s.m & 56 & 92,9 & 0,95 & $(0,87,1,03)$ & 52 & 84,6 & 0,99 & $(0,84,1,17)$ \\
\hline 1 a 3 s.m & 33 & 97 & 1,03 & $(0,94,1,11)$ & 32 & 81,3 & 0,94 & $(0,77,1,13)$ \\
\hline$>3 \mathrm{~s} . \mathrm{m}$ & 17 & 100 & & & 16 & 93,8 & & \\
\hline
\end{tabular}

RP (Razão de prevalência); IC 95\% (Intervalo de confiança) *p<0,05 
Tabela 3. Fatores sociodemográficos associados à categoria crenças sobre benefícios em relação ao estresse. Salvador, Bahia, 2006. $(n=106)$

\begin{tabular}{|c|c|c|c|c|c|c|c|c|c|c|}
\hline \multirow[b]{3}{*}{ Variáveis } & \multicolumn{10}{|c|}{ Estresse } \\
\hline & \multicolumn{3}{|c|}{ Evitar preocupação } & \multicolumn{4}{|c|}{ Ter horas delazer e diversão } & \multicolumn{3}{|c|}{ Fazer relaxamento } \\
\hline & $\mathrm{N}$ & $\%$ & IC95\% & $\mathrm{N}$ & $\%$ & RP & IC95\% & $\mathrm{N}$ & $\%$ & IC95\% \\
\hline \multicolumn{11}{|l|}{ Gênero } \\
\hline Homens & 28 & 21,4 & $1,39(0,58,3,36)$ & 28 & 78,6 & 1,25 & $(0,97,1,62)$ & 28 & 71,4 & $1,14(0,85,1,52)$ \\
\hline Mulheres & 78 & 15,4 & & 78 & 62,8 & & & 78 & 62,8 & \\
\hline \multicolumn{11}{|l|}{ Idade(anos) } \\
\hline$<45$ & 26 & 11,5 & $0,61(0,19,1,96)$ & 26 & 80,8 & 1,29 & $(1,00,1,66)$ & 26 & 76,9 & $1,26(0,96,1,65)$ \\
\hline $45-54$ & 26 & 23,1 & $1,54(0,64,3,69)$ & 26 & 80,8 & 1,29 & $(1,00,1,66)$ & 26 & 61,5 & $0,92(0,66,1,30)$ \\
\hline$>=54$ & 54 & 16,7 & & 54 & 53,7 & & & 54 & 61,1 & \\
\hline \multicolumn{11}{|l|}{ Estado civil } \\
\hline Sem companheiro & 65 & 16,9 & $0,99(0,42,2,35)$ & 65 & 66,2 & 0,97 & $(0,74,1,27)$ & 65 & 64,6 & $0,98(0,74,1,30)$ \\
\hline Com companheiro & 41 & 17,1 & & 41 & 68,3 & & & 41 & 65,9 & \\
\hline \multicolumn{11}{|l|}{ Escolaridade } \\
\hline A té $1^{0}$ grau & 67 & 13,4 & $0,58(0,25,1,34)$ & 67 & 62,7 & 0,84 & $(0,65,1,09)$ & 67 & 56,7 & $0,71(0,55,0,93)$ \\
\hline $2^{0}$ grau & 32 & 25 & $1,85(0,80,4,25)$ & 32 & 71,9 & 1,1 & $(0,84,1,46)$ & 32 & 78,1 & $1,31(1,01,1,71)$ \\
\hline 3o grau & 7 & 14,3 & & 7 & 85,7 & & & 7 & 85,7 & \\
\hline \multicolumn{11}{|l|}{ Renda } \\
\hline$<1$ s.m & 56 & 14,3 & $0,71(0,31,1,67)$ & 56 & 62,5 & 0,87 & $(0,66,1,13)$ & 56 & 60,7 & $0,87(0,66,1,14)$ \\
\hline 1 a 3 s.m & 33 & 12,1 & $0,63(0,22,1,77)$ & 33 & 69,7 & 1,06 & $(0,80,1,40)$ & 33 & 60,6 & $0,9 \quad(0,66,1,24)$ \\
\hline$>3 \mathrm{s.m}$ & 17 & 35,3 & & 17 & 76,5 & & & 17 & 88,2 & \\
\hline
\end{tabular}

RP (Razão de prevalência); IC 95\% (Intervalo de confiança) *p<0,05

Tabela 4. Fatores sociodemográficos associados à categoria crenças sobre benefícios em relação a álcool e fumo. Salvador, Bahia, 2006. ( $n=106)$

\begin{tabular}{|c|c|c|c|c|c|c|c|c|}
\hline \multirow[b]{3}{*}{ Variáveis } & \multicolumn{8}{|c|}{ Bebida al coólica e fumo } \\
\hline & \multicolumn{4}{|c|}{ Evitar o consumo de álcool } & \multicolumn{4}{|c|}{ Não fumar ou parar de fumar } \\
\hline & $\mathrm{N}$ & $\%$ & $\mathrm{RP}$ & IC95\% & $\mathrm{N}$ & $\%$ & $\mathrm{RP}$ & IC95\% \\
\hline \multicolumn{9}{|l|}{ Gênero } \\
\hline Homens & 26 & 76,9 & 0,83 & $(0,67,1,04)$ & 26 & 96,2 & 0,99 & $(0,91,1,07)$ \\
\hline M ulheres & 76 & 92,1 & & & 77 & 97,4 & & \\
\hline \multicolumn{9}{|l|}{ Idade(anos) } \\
\hline$<45$ & 25 & 84 & 0,94 & $(0,78,1,13)$ & 25 & 96 & 0,98 & $(0,90,1,08)$ \\
\hline $45-54$ & 26 & 88,5 & 1 & $(0,85,1,18)$ & 25 & 96,2 & 0,99 & $(0,91,1,07)$ \\
\hline$>=54$ & 51 & 90,2 & & & 52 & 98,1 & & \\
\hline \multicolumn{9}{|l|}{ Estado civil } \\
\hline Sem companheiro & 62 & 87,1 & 0,97 & $(0,84,1,11)$ & 62 & 96,8 & 0,99 & $(0,93,1,06)$ \\
\hline Com companheiro & 40 & 90 & & & 41 & 97,6 & & \\
\hline \multicolumn{9}{|l|}{ Escolaridade } \\
\hline Até $1^{0}$ grau & 63 & 88,9 & 1,02 & $(0,88,1,18)$ & 65 & 96,9 & 0,99 & $(0,93,1,06)$ \\
\hline $2^{\circ}$ grau & 32 & 87,5 & 0,99 & $(0,84,1,15)$ & 31 & 96,8 & 0,99 & $(0,92,1,07)$ \\
\hline 30 grau & 7 & 85,7 & & & 7 & 100 & & \\
\hline \multicolumn{9}{|l|}{ Renda } \\
\hline$<1$ s.m & 54 & 90,7 & 1,06 & $(0,92,1,23)$ & 55 & 96,4 & 0,98 & $(0,92,1,05)$ \\
\hline 1 a 3 s.m & 31 & 90,3 & 1,03 & $(0,89,1,20)$ & 31 & 100 & 1,04 & $(0,99,1,09)$ \\
\hline$>3 \mathrm{s.m}$ & 17 & 76,5 & & & 17 & 94,1 & & \\
\hline
\end{tabular}

RP (Razão de prevalência); IC95\% (Intervalo de confiança) *p<0,05 


\begin{tabular}{|c|c|c|c|c|c|c|c|c|c|}
\hline \multicolumn{10}{|c|}{ 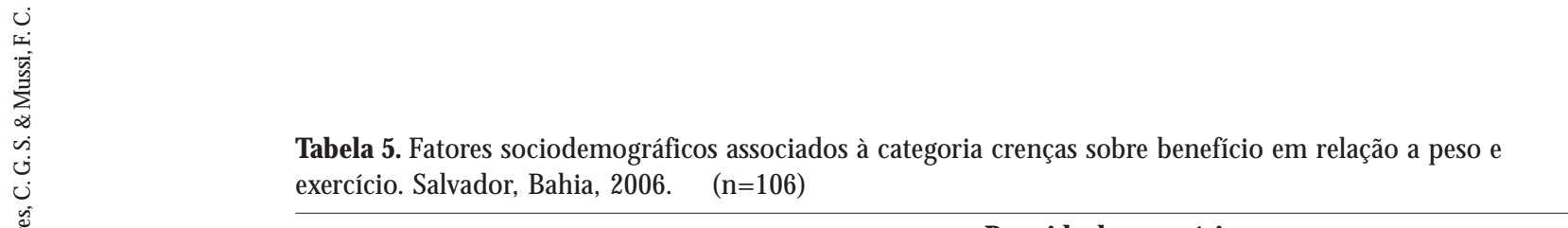 } \\
\hline & \multirow[b]{3}{*}{ Variáveis } & \multicolumn{8}{|c|}{ Peso ideal e exercício } \\
\hline & & \multicolumn{4}{|c|}{ Manter o peso ideal } & \multicolumn{4}{|c|}{ Praticar exercício físico } \\
\hline & & $\mathrm{N}$ & $\%$ & $\mathrm{RP}$ & IC95\% & $\mathrm{N}$ & $\%$ & $\mathrm{RP}$ & IC $95 \%$ \\
\hline & \multicolumn{9}{|l|}{ Gênero } \\
\hline & Homens & 28 & 100 & 1,05 & $(1,00,1,11)$ & 25 & 100 & 1,09 & $(1,02,1,17)$ \\
\hline & \multicolumn{9}{|l|}{ Idade(anos) } \\
\hline & $<45$ & 26 & 92,3 & 0,95 & $(0,84,1,06)$ & 26 & 96,2 & 1,04 & $(0,94,1,15)$ \\
\hline & $45-54$ & 26 & 96,2 & 1 & $(0,91,1,09)$ & 26 & 92,3 & 0,98 & $(0,86,1,11)$ \\
\hline & $>=54$ & 54 & 98,1 & & & 43 & 93 & & \\
\hline & \multicolumn{9}{|l|}{ Estado civil } \\
\hline & Sem companheiro & 65 & 93,8 & 0,94 & $(0,88,1,00)$ & 59 & 94,9 & 1,03 & $(0,92,1,16)$ \\
\hline & Com companheiro & 41 & 100 & & & 36 & 91,7 & & \\
\hline & \multicolumn{9}{|l|}{ Escolaridade } \\
\hline & Até $1^{\circ}$ grau & 67 & 98,5 & 1,07 & $(0,97,1,17)$ & 57 & 91,2 & 0,94 & $(0,85,1,03)$ \\
\hline & $2^{\circ}$ grau & 32 & 90,6 & 0,92 & $(0,82,1,03)$ & 32 & 96,9 & 1,05 & $(0,96,1,16)$ \\
\hline & 3o grau & 7 & 100 & & & 6 & 100 & & \\
\hline & \multicolumn{9}{|l|}{ Renda } \\
\hline & $<1$ s.m & 56 & 94,6 & 0,97 & $(0,90,1,04)$ & 49 & 93,9 & 1 & $(0,90,1,11)$ \\
\hline & 1 a $3 \mathrm{s.m}$ & 33 & 100 & 1,06 & $(1,00,1,12)$ & 30 & 90 & 0,94 & $(0,83,1,07)$ \\
\hline & $>3 \mathrm{s.m}$ & 17 & 94,1 & & & 16 & 100 & & \\
\hline
\end{tabular}

RP (Razão de prevalência); IC95\% (Intervalo de confiança) *p<0,05

As pessoas sem companheiros, em relação às com companheiros, perceberam mais crenças sobre benefícios apenas para evitar gordura e praticar exercícios, havendo diferença estatisticamentesignificantepara consultar o médico uma vez por ano. Aqueles que recebiam menos que um salário mínimo, em relação aos com renda mensal superior a três salários mínimos, perceberam menos benefícios para nove dos doze comportamentos de saúde, exceto para evitar 0 consumo de álcool, havendo diferença estatisticamente significante para consumo de comida com menos gordura.

Esses resultados mostraram tendência à menor percepção de crenças em saúde sobre ben efícios para pessoas mais jovens, sem companheiro, com baixa escolaridade e renda e diferenças de gênero na percepção da natureza das crenças sobre benefícios.

\section{Discussão}

No presente estudo, os 106 adultos negros e hipertensos foram caracterizados, predominante- mente, por mulheres, com idade mediana de 54 anos, com baixa escolaridadeerenda mensal, por não ter companheiro e exercer atividade laboral.

Embora a percepção de ben efícios quanto à adoção de medidas de prevenção e controle da hipertensão arterial não impliquenecessariamente a coerência e efetividade da ação, a crença é, por si só, é um forte preditor do comportamento. Assim, o predomínio da categoria "crenças em saúde sobre benefícios" parece constituir-se em um importante indicador para a adesão. De acordo com o Modelo de Crenças em Saúde, a pessoa, para mudar comportamentos de saúde, necessita primeiramente valorizar os benefícios das mudanças de estilo de vida, ou seja, acreditar queépossível reduzir a suscetibilidade ou severidade da doença.

0 fato de $100 \%$ dos participantes terem percebido benefícios para a medida da pressão arterial revelou que este é um parâmetro valorizado para indicar o controle da hipertensão arterial. Defato, recomenda-se que essa aferição seja realizada em todas as consultas médicas, independenteda especialidade, pois setrata de um procedimento defácil execução e, se realizado correta- 
mente, é uma das maneiras mais rápidas, seguras e eficazes de se detectar a doença ${ }^{13}$.

Ainda, 30,2\% da amostra perceberam barreiras para usar menos sal na comida, pois a re dução implicava fazer esforço, ter menos prazer e estar envolvido no preparo do alimento. Talvez essas barreiras possam ser minimizadas se as pessoas forem orientadas a tornar a comida mais palatável, utilizando outros elementos culináriOS, a exemplo do uso de mais limão, vinagre e azeite doce em vez do sal. Vale ressaltar que a culinária nordestina, mais especificamente a baiana, érica em pratos tradicionais preparados com muito sal, gordura e condimentos. Assim, as pessoas que têm hipertensão arterial, como o grupo entrevistado neste estudo, necessitam também da ajuda de amigos e familiares para manter a prática saudável de reduzir o sal. Sabe-sequea redução da ingestão do sal na dieta, além da redução da pressão arterial, determina a queda da mortalidade por acidente vascular encefálico e a regressão da hipertrofia ventricular esquerda ${ }^{14}$. Outros autores também constataram a dificuldade para diminuição do consumo de sal ${ }^{15}$.

Chamou também a atenção que $63,2 \%$ dos participantes ficaram indecisos quanto ao benefício de evitar preocupação para o controle da hipertensão arterial. Todavia, há que se destacar que parece difícil um indivíduo querer se afastar, evitar ou ocultar o que talvez considere como intrínseco à vida, uma preocupação vital ${ }^{16}$.

Considerando que as mudanças comportamentais representam o abandono de alguns prazeres, em especial para pessoas que lutam para sobreviver em condições de vida de pobreza, é sempre necessário que a educação em saúde se faça de modo a contemplar a individual idade e 0 contexto social da pessoa. É importante também que os educadores em saúde, incluindo a enfermeira, exerçam a função de encorajar os indivíduos hipertensos a assumir a responsabilidade de proteção da saúde, de ajudar a encontrar, no âmbito das suas possibilidades, a adoção de hábitos de vida saudáveis, no intuito de promover o controle e a prevenção de agravos da doença.

Como se pôde perceber, a análise das associações entre fatores sociodemográficos e 0 indicador de crenças em saúde mostrou que a baixa escolaridade e a renda e que pessoas em idades mais jovens e sem companheiro perceberam menos ben efícios das medidas de controle da hipertensão arterial, corroborando a afirmação de vários autores de que as variáveis sociodemográficas são preditoras de adesão ao tratamento anti-hipertensivo ${ }^{17-21}$.
Embora homens emulheres tenham percebido diferentementemais ou menos benefícios para os doze comportamentos de saúde, a diferença não foi estatisticamentesignificantepara os distintos comportamentos. Talvez a diferença nesta percepção possa estar associada ao gênero. Homens e mulheres conceituam e expressam as suas questões de saúde deformas distintas, como resultado de experiências pessoais e da variabilidade social de que são herdeiros e em que se desenvolvem, principalmente. Segundo Sousa9, tabagismo, alcoolismo e abuso de drogas são mais comuns entrehomens eeles demonstraram menor crença em saúde sobre benefícios quanto a essas variáveis. Alguns estudos mostraram maior aderência a esferas do tratamento por mulheres, 9,20 .

As pessoas com menos de 45 anos demonstraram mais crenças sobre benefícios, quando comparadas àquelas na faixa etária $>=a 54$ anos, relacionando-os a apenas fazer relaxamento, ter horas de diversão elazer epraticar exercícios físicos. Com relação à idade, outros autores também constataram que pessoas de idade mais avançada são mais propensas a adesão ${ }^{19,22,23}$. Esses achados indicam que o jovem não se sente vulnerável à doença, enquanto os mais velhos, mais preocupados com a saúde, apegam-se ao tratamento como alternativa de prolongamento da vida ${ }^{23}$.

As pessoas com 1 음au, em relação às com 30 grau, perceberam maior benefício apenas para manter o peso ideal e evitar o consumo deálcool. Gigante, Barros e $\mathrm{Post}^{24}$ constataram que as pessoas com maior escolaridade foram significativamente menos obesas. Outros autores evidenciaram que pessoas hipertensas com maior nível de escolaridade eram mais aderentes ao tratamento ${ }^{19-22}$. Deficiências na formação escolar podem trazer dificuldades relativas à assimilação de orientações dispensadas pelos profissionais e influenciar na percepção da gravidade da doença ${ }^{25}$. Por outro lado, a baixa escolaridade precisa ser considerada na escol ha de estratégias para orientação e abordagem nas consultas individuais.

Pessoas sem companheiros, em relação às com companheiros, perceberam apenas maior ben efício para evitar gordura e praticar exercícios. Chor ${ }^{18}$ verificou que pessoas com companheiros apresentavam chance duas vezes maior de estarem realizando o tratamento em relação àquelas sem companheiros. 0 estado civil foi também apontado em outros estudos como antecedente da adesão ao tratamento da hipertensão arterial19,20,22. Isso pode ser justificável se for considerado 0 acolhimento familiar, a possibili- 
dade de incentivo do companheiro para fazer dieta, exercício físico, tomar remédios, entre outros fatores.

As pessoas que recebiam menos que um salário mínimo, em relação às com renda mensal superior a três salários mínimos, perceberam maior benefício apenas para evitar o consumo deálcool. No que se refere à renda, al guns autores mencionaram que este fator está diretamente relacionado à adesão ${ }^{20,23}$. 0 status econômico influencia sobremodo na habilidade das pessoas em seguir as recomendações não só no que se relaciona à compra de medicamentos e alimentos adequados, a freqüentar uma academia, ter lazer e diversão, mas também a fatores ligados aos aspectos educacionais, culturais e sociais. Contrariamente, pode ser elemento de estresse se a renda for insuficiente para a subsistência básica ${ }^{20,23}$. A Organização Mundial de Saúde informa quea não adesão ao tratamento da hipertensão arterial está relacionada ao baixo poder aquisitivo, analfabetismo, desemprego, fornecimento de medicamentos limitado e o alto custo desses. Assim sendo, as intervenções envolvendo tais temáticas deveriam abarcar suporte previdenciário ou seguro-saúde às pessoas hipertensas, não interrupção do suprimento medicamentoso e poder aquisitivo individual suficiente, capaz de arcar com um sistema de suprimento seguro das diversas necessidades apresentadas no decorrer do tratamento ${ }^{26}$.

Sea mudança de estilo devida, no que se refere ao tratamento da hipertensão arterial, é por si só difícil devido a restrições a prazeres como comer o que se quer e se gosta, não fumar, não be ber e/ou a incorporação de hábitos não apreciados, como a prática de atividade física, controle médico, uso de medicamentos, dentre outros, parece mais penosa essa mudança quando o status econômico e social é também precário, porque as possibilidades de aproximação dos desejos pessoais às medidas terapêuticas são mais limitadas. Assim, talvez, variáveis relacionadas a barreiras sociais eeconômicas possam implicar a percepção favorável ou desfavorável para a adoção das medidas de prevenção e controle da doença.

\section{Consideraçõesfinais}

A análise global sobre as crenças quanto às medidas de prevenção e controle da hipertensão arterial mostrou predomínio da categoria crenças sobre benefícios e tendência à percepção de menor crença sobre benefícios em estratos socioeconômicos menos favorecidos, adultos jovens e pessoas sem companheiro. 0 estudo contribuiu para a identificação de grupos de risco e indicadores de adesão ao tratamento e para a reflexão da importância de um cuidar que reconheça a subjetividade, o quesito raça/cor, o gênero, a geração ea classe socioeconômica como fatores que podem fortemente influenciar na adesão. Concorreu também para alertar os profissionais de saúde sobre a importância desses achados relativamente às considerações e adoção de estratégias educativas.

Chama-se a atenção para a importância de outros estudos que comparem os resultados desta pesquisa, cujo foco privilegiepopulações de outros grupos étnicos/raciais, como forma de verificar sea cor/raça éum fator de influência na percepção das crenças em saúde. Entende-se ainda que, sem compreender como as pessoas pensam e se sentem diante dos seus problemas de saúde, não sepode promover, quiçá concretizar um processo educativo quevislumbrea mudança ea sustentação deum estilo devida saudável. Finalmente, é necessário e possível investir todo o esforço para incentivar e permitir a participação ativa da pessoa no seu processo desaúde/doença por meio de uma abordagem interdisciplinar. 


\section{Colaboradores}

CGS Pires e FC Mussi participaram igualmente de todas as etapas da elaboração do artigo.

\section{Referências}

1. Ide CAC. Atenção ao doente crônico: um sistema teórico-instrumental em ressignificação. In: Pierin AM G, organizadora. Hipertensão arterial - uma proposta para o cuidar. Barueri, SP: M anole; 2004. p. 1-9.

2. Pierin $A M G$. Adesão ao tratamento: conceitos. In: Nobre F, Pierin AM G, Mion Jr D, organizadores. Adesão ao tratamento: 0 grande desafio da hipertensão. São Paulo: Lemos Editorial; 2001. p. 21-33.

3. Pierin AM G, Strelec MAAM, Mion Jr D. 0 desafio do controle da hipertensão arterial e a adesão ao tratamento. In: Pierin AMG, organizadora. Hipertensão arterial - uma proposta para o cuidar. Barueri, SP: M anole; 2004. p. 274-289.

4. Araújo GBS. Adesão ao tratamento anti-hipertensivo: análise conceitual [dissertação]. João Pessoa (PB): Centro de Ciências da Saúde; 2002.

5. Rodrigues MTM. Caminhos e descaminhos da adesão ao tratamento anti- hipertensivo: um estudo com usuários do PACHA do Hospital Universitário O nofre Lopes [dissertação]. Natal (RN): Centro de Ciências Humanas, Letras e Artes; 2003.

6. Gusmão JL, Mion Jr D. Adesão ao tratamento conceitos. Rev Bras Hipertens 2006; 13(1): 23-25.

7. Vieira VA. Hipertensão arterial e aspectos éticos em pesquisa envolvendo seres humanos: implicações para a área da saúde. Rev Bras Saúde M ater Infant 2003; 3(4):481-488.

8. Dela Coleta M F. 0 modelo de crenças em saúde: uma aplicação a comportamentos de prevenção e controle da doença [tese]. Brasília: Instituto de Psicologia; 1995.

9. Sousa ALL. Prevalência da hipertensão arterial referida, percepção de sua origem e formas de controle em área metropolitana de São Paulo [tese]. São Paulo (SP): Faculdade de Saúde Pública; 1999.

10. Instituto Brasileiro e geografia e Estatística. Resultados da Amostra do Censo Demográfico 2000. Área de ponderação Liberdade, Sieiro e Japão. [acessado 2006 nov 1]. Disponível em: http://www.ibge. gov.br/home/estatistica/populacao/censo 2000

11. Rosner B. Fundamentals of biostatistics. $4^{\text {th }}$ ed. Boston: Duxbury Press; 1995.

12. Brasil. Ministério da Saúde. Resolução n 196/96. Sobre a pesquisa envolvendo seres humanos. Braślia: Ministério da Saúde; 1996.

13. Alavarce DC, Pierin AMG, Mion Jr D. A pressão arterial está sendo medida? Rev. Esc. Enf. USP 2000; 34(1): 84-90.
14. Salgado CM, Carvalhaes JTA. Hipertensão arterial na infância. J. Pediatr. 2003; 79(1):115-124.

15. Lima TM, Bucher JSNF, Oliveira Lima, JW. A hipertensão arterial sob o olhar de uma população carente: estudo exploratório a partir dos conhecimentos, atitudes e práticas. Cad Saúde Pública 2004; 20(4):1079-1087.

16. Gandarilhas MA, Câmara SG, Scarparo H. Estressores sociais da hipertensão em comunidades carentes. Psicol. Reflex. Crit. 2005; 18(1):62-71.

17. Sadala M LA, M endes IM. Vivenciando a hipertensão. Revista da Sociedade de Cardiologia do Estado de São Paulo 1996; 6(3):1-6.

18. Chor D. Hipertensão arterial entre funcionários de banco estatal no Rio de Janeiro. Hábitos de vida e tratamento. Arq. Bras. Cardiol 1998; 71(5):653-660.

19. Kungäs $H$, Lahdenperä $T$. Compliance of patients with hypertension and associated factors. Journal of Advanced Nursing 1999; 29(4):832-839.

20. Clark MJ, Curran C, Noji A. The effects of community health nurse monitoring on hypertension identification and control. Public Health Nursing 2000; 17(6):452-459.

21. V Diretrizes Brasileiras de Hipertensão Arterial. Revista da Sociedade Brasileira de Hipertensão 2006, 6(5):1-48.

22. M oreira TM M, Araújo TL. Falta de adesão ao tratamento da hipertensão: características de uma clientela distinta. Rev. RENE 2001; 2(2):39-46.

23. Car MR, Pierin AMG, AquinoVLA. Estudo sobre a influência do processo educativo no controle da hipertensão arterial. Rev. Esc. Enf. USP 1991; 25(3):255-269.

24. Gigante DP, Barros FC, Post CLA. Prevalence and risk factors of obesity in adults. Rev. Saúde Pública 1997; 31(3):236-246.

25. Guedes N, Costa FBC, Moreira RP, Moreira TF, Chaves ES, Araújo TL de. Crises hipertensivas em portadores de hipertensão arterial em tratamento ambulatorial. Rev Esc Enf USP 2005, 39(2):181-188.

26. Sabaté $\mathrm{E}$. Adherence to long-term therapies: evidence for action. Geneve: World Health Organization; 2003.

Aprovado em 27/12/2006

Versão final apresentada em 01/11//2007 